DOI:

УДК [519.876.5:530.182]:553.98

А.М. Сінчук, к.т.н., sinchuklm@ukr.net

Рівненський державний гуманітарний університет, м. Рівне

\title{
МАТЕМАТИЧНЕ МОДЕЛЮВАННЯ ДВОФАЗНОЇ ФІЛЬТРАЦІЇ В АНІЗОТРОПНИХ НАФТОВИХ ПЛАСТАХ МЕТОДОМ КВАЗІКОНФОРМНОГО ВІДОБРАЖЕННЯ
}

У даній роботі метод комплексного аналізу нелінійних процесів витіснення флюїдів модифіковано на випадок моделювання нелінійної фільтраиії в анізотропних середовищах. 3 використанням системи типу Коші-Рімана (у випадку врахування анізотропії), побудовано нові умови для визначення положення граничних вузлів гідродинамічної сітки відносно приграничних. 3 урахуванням ивого було модифіковано відповідний алгоритм числового квазіконформного відображення та здійснено його програмну реалізаиію. Проведено ряд комп'ютерних експериментів впливу зміни компонент тензора проникності на напрямок течії в елементі симетрій рядної системи пломового заводнення пласта.

Ключові слова: нафтовидобування; анізотропні середовища; числові методи квазіконформних відображень; нелінійні задач.

In this paper a complex method of investigation for nonlinear processes of fluid driving is modified for a case, when a problem of nonlinear filtration modeling in anisotropic media. Using the Cauchy-Riemann-type system (in the case of anisotropy), new conditions have been constructed to determine the position of the boundary nodes of the hydrodynamic grid relatively to cross-border ones. In view of this, the corresponding algorithm of the numerical quasi-conformal mapping was modified and its software implementation was realized. A series of computer experiments on the effect of changing the permeability tensor components on the direction flows in the symmetry element of the in-line system of the layer flooding was carried out.

Key words: oil production; anisotropic media; the numerical methods of quaziconformal mappings; nonlinear problems.

\section{Постановка проблеми}

За останні десятиріччя відбувається погіршення процесу нафтовидобутку. Головним чином це пов'язане із структурою порід-колекторів, більша половина 3 яких становить важковидобувні, енергетично-виснажені та високообводнені поклади. Їх висока неоднорідність призводить, зокрема, до нерівномірного вилучення нафти, зниження коефіцієнта нафтовіддачі та ін.

Більшість існуючих моделей описують процеси фільтрації за умов ізотропії пласта, коли коефіцієнт проникності є скалярною функцією $[1,2]$. Проте, на практиці зустрічаються пласти, коефіцієнт проникності яких залежить від напрямку фільтрації, тобто градієнт тиску є не колінеарним до вектору швидкості. У таких випадках має місце явище анізотропії пласта [3-5], а закон Дарсі, при сумісному русі декількох рідин, набуває вигляду:

$$
\vec{v}_{i}=-\left(\begin{array}{lll}
k_{11} & k_{12} & k_{13} \\
k_{21} & k_{22} & k_{23} \\
k_{31} & k_{32} & k_{33}
\end{array}\right) \frac{\tilde{k}_{i}\left(s_{i}\right)}{\mu_{i}} \operatorname{gradp}, i=\overline{1, l} .
$$

В зв'язку з чим виникає потреба узагальнення розроблених нами, зокрема в працях [6-8], методів комплексного аналізу на випадок урахування анізотропії пласта.

Аналіз останніх досліджень та публікацій

Розвитком численних розділів підземної гідромеханіки, які охоплюють теорію фільтрації рідин у викривлених нескінченно тонких шарах постійної та змінної товщини, займались такі вчені, як Баснієв К. С., Кочіна I. М., Максімов В. М., Баренблатт Г.І., Снтов В. М., Рижик В. М., тощо $[1,2]$. Вони досліджували нелінійні процеси фільтрації різного роду рідин в ізотропних середовищах. Однак, з кінця XX століття і по теперішній час, дослідження нелінійних процесів витіснення нафти в анізотропних середовищах набуває все більшої популярності. Зок- 
рема, такі вчені, як Пергамент А. Х., Томін П. Ю., Толпаєв В.А., Мартинов В.В., Євенко І.А. в своїх роботах $[3,4]$ присвятили велику увагу дослідженню та розвитку методів математичного моделювання відповідних процесів. Низка робіт Бомби А. Я., Каштана С. С., Пригорницького Д. О., Ярощака С. В. присвячені розвитку чисельних методів розв'язування двовимірних задач фільтрації методами конформного, квазіконформного відображень та поетапної фіксації характеристик середовища й процесу. Зокрема у [6], авторами продемонстровано розвиток методів комплексного аналізу для математичного моделювання та оптимізації параметрів фільтраційних процесів з урахуванням зворотного впливу визначальних факторів процесу на характеристики середовища.

\section{Формулювання мети дослідження}

Метою даної роботи є створення математичної моделі нелінійного процесу витіснення в анізотропних нафтових пластах та розробка ефективного методу чисельного розв'язання відповідної задачі.

\section{Виклад основного матеріалу}

Розглядається модельна задача двофазної ізотермічної фільтрації в елементі симетрії рядної системи заводнення (рис. 1) анізотропного пласта [5]. Контури області фільтрації $G_{z}$ $(z=x+i y)$ задаємо наступними співвідношеннями:

$$
\begin{gathered}
A B=\left\{z: f_{1}(x, y)=0\right\}=\left\{z: x=r \cos \alpha, y=r \sin \alpha+b,-\frac{\pi}{2} \leq \alpha \leq \frac{\pi}{2}\right\}, \\
E F=\left\{z: f_{3}(x, y)=0\right\}=\left\{z: x=r \cos \alpha+h, y=r \sin \alpha+b, \frac{\pi}{2} \leq \alpha \leq \frac{3 \pi}{2}\right\}, \\
B C D E=\left\{z: f_{2}(x, y)=0\right\}=B C \cup C D \cup D E, \\
F G H A=\left\{z: f_{4}(x, y)=0\right\}=F G \cup G H \cup H A, \\
B C=\{z: x=0, b+r \leq y \leq 2 b\}, C D=\{z: 0 \leq x \leq h, y=2 b\}, \\
D E=\{z: x=h, b+r \leq y \leq 2 b\}, \\
F G=\{z: x=h, 0 \leq y \leq a\}, H A=\{x=0,0 \leq y \leq a\}, \\
G H=\{z: 0 \leq x \leq h, y=0\},
\end{gathered}
$$

де $a=|H A|=|B C|=|D E|=|F G|, b=a+r, r-$ радіус свердловин, $h$ - відстань між центрами свердловин.

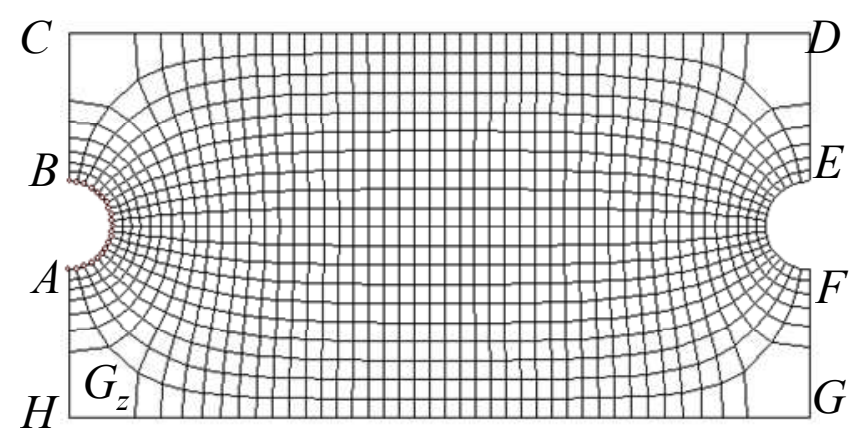

Рис. 1. Елемент симетрії рядної системи заводнення

Закон руху та рівняння нерозривності течії, записані відносно квазіпотенціалу швидкості фільтрації $\varphi=\varphi(x, y, t)$, згідно з $[4,6]$ подамо у вигляді

$$
\frac{\partial\left(\sigma s_{l}\right)}{\partial t}+\operatorname{div}\left(v_{l}\right)=0,(l=o, w)
$$




$$
\vec{v}_{l}=\left(\begin{array}{ll}
k_{11} & k_{12} \\
k_{21} & k_{22}
\end{array}\right) \frac{\tilde{k}_{l}(s)}{\mu_{l}} \operatorname{grad} \varphi,(l=o, w), \quad s_{o}+s_{w}=1,
$$

де $\mu_{l}$ - динамічна в'язкість, $\vec{v}_{l}$ - швидкість фільтрації $l$-ї фази, $s_{l}=s_{l}(x, y, t)-$ насиченість пласта $l$-ю фазою, $t$ - час; $\sigma=$ const,$k=\left(\begin{array}{ll}k_{11} & k_{12} \\ k_{21} & k_{22}\end{array}\right)$ - коефіцієнти пористості та абсолютної проникності грунту, $k_{r s}=$ const $(r, s=1,2)$ - величини, що характеризують проникність пласта та його анізотропію [2,3], $\tilde{k}_{w}=\tilde{k}_{w}(s), \tilde{k}_{o}=\tilde{k}_{o}(s)-$ відносні фазові проникності $\left(s=s_{w}\right)$. Індексами « $w$ » « $O »$ позначено величини, що характеризують воду та нафту. Звідси, з урахуванням сумарної швидкості $\vec{v}=\vec{v}_{w}+\vec{v}_{o}$ фільтраційної течії, маємо:

$$
\begin{gathered}
\operatorname{div} \vec{v}=0, \\
\vec{v}=\left(\begin{array}{ll}
k_{11} & k_{12} \\
k_{21} & k_{22}
\end{array}\right)\left(\frac{\tilde{k}_{o}(s)}{\mu_{o}}+\frac{\tilde{k}_{w}(s)}{\mu_{w}}\right) \operatorname{grad} \varphi, \\
\sigma \frac{\partial s}{\partial t}+\vec{v} \cdot \operatorname{grad} f(s)=0, \\
s(x, y, t)=\left.\tilde{s}(x, y)\right|_{t=0},\left.s\right|_{A B}=s_{0},
\end{gathered}
$$

де $f(s)=\frac{\mu_{1} \tilde{k}_{w}(s)}{\mu_{w} \tilde{k}_{o}(s)+\mu_{o} \tilde{k}_{w}(s)}, \tilde{s}(x, y), s_{0}$ - функція, що задає початковий розподіл насиченості в пласті і їі значення на нагнітальній свердловині. Задачу фільтрації розв'язуватимемо при наступних крайових умовах: $\left.\varphi\right|_{A B}=\varphi_{*},\left.\varphi\right|_{E F}=\varphi^{*},\left.\frac{d \varphi}{d x}\right|_{B C}=0,\left.\frac{d \varphi}{d y}\right|_{C D}=0,\left.\frac{d \varphi}{d x}\right|_{D E}=0,\left.\frac{d \varphi}{d x}\right|_{F G}=0$, $\left.\frac{d \varphi}{d y}\right|_{G H}=0,\left.\frac{d \varphi}{d x}\right|_{H A}=0$.

Введемо функцію $\psi$, комплексно спряжену до $\varphi$, та запишемо задачу $(1-4)$ у більш зручному, для використання методу квазіконформного відображення [6], вигляді:

$$
\begin{gathered}
\frac{\partial \psi}{\partial y}=\bar{k}\left(k_{11} \frac{\partial \varphi}{\partial x}+k_{12} \frac{\partial \varphi}{\partial y}\right),-\frac{\partial \psi}{\partial x}=\bar{k}\left(k_{21} \frac{\partial \varphi}{\partial x}+k_{22} \frac{\partial \varphi}{\partial y}\right), \\
\frac{\partial s}{\partial t}=-\frac{\bar{k}}{\sigma}\left(\frac{\partial f}{\partial x}\left(k_{11} \frac{\partial \varphi}{\partial x}+k_{12} \frac{\partial \varphi}{\partial y}\right)+\frac{\partial f}{\partial y}\left(k_{21} \frac{\partial \varphi}{\partial x}+k_{22} \frac{\partial \varphi}{\partial y}\right)\right), \\
\left.\varphi\right|_{A B}=\varphi_{*},\left.\varphi\right|_{E F}=\varphi^{*},\left.\psi\right|_{A H G F}=0,\left.\psi\right|_{B C D E}=Q, \\
s(x, y, t)=\left.\tilde{s}(x, y)\right|_{t=0},\left.s\right|_{A B}=s_{0}, \\
\text { де } Q=\int_{A B} \bar{k}\left(k_{11} \frac{\partial \varphi}{\partial x}+k_{12} \frac{\partial \varphi}{\partial y}\right) d y-\bar{k}\left(k_{21} \frac{\partial \varphi}{\partial x}+k_{22} \frac{\partial \varphi}{\partial y}\right) d x, \bar{k}=\frac{\tilde{k}_{o}(s)}{\mu_{o}}+\frac{\tilde{k}_{w}(s)}{\mu_{w}} . \\
\text { Обернена до (5-6) задача має вигляд: } \\
\frac{\partial x}{\partial \varphi}=\bar{k}\left(k_{11} \frac{\partial y}{\partial \psi}-k_{12} \frac{\partial x}{\partial \psi}\right), \quad \frac{\partial y}{\partial \varphi}=\bar{k}\left(k_{21} \frac{\partial y}{\partial \psi}-k_{22} \frac{\partial x}{\partial \psi}\right), \\
x\left(\varphi_{*}, \psi\right)= \\
r \cos \alpha, y\left(\varphi_{*}, \psi\right)=r \sin \alpha+b,-\frac{\pi}{2} \leq \alpha \leq \frac{\pi}{2}, 0 \leq \psi \leq Q, \\
x(\varphi, Q)=0, b+r \leq y(\varphi, Q) \leq 2 b, \varphi_{*} \leq \varphi \leq \varphi_{C}, \\
0 \leq x(\varphi, Q) \leq h, y(\varphi, Q)=2 b, \varphi_{C} \leq \varphi \leq \varphi_{D}, \\
x(\varphi, Q)=h, b+r \leq y(\varphi, Q) \leq 2 b, \varphi_{D} \leq \varphi \leq \varphi^{*},
\end{gathered}
$$




$$
\begin{gathered}
x\left(\varphi^{*}, \psi\right)=r \cos \alpha+h, y\left(\varphi^{*}, \psi\right)=r \sin \alpha+b, \frac{\pi}{2} \leq \alpha \leq \frac{3 \pi}{2}, 0 \leq \psi \leq Q, \\
x(\varphi, 0)=h, 0 \leq y(\varphi, 0) \leq a, \varphi_{G} \leq \varphi \leq \varphi^{*}, \\
0 \leq x(\varphi, 0) \leq h, y(\varphi, 0)=0, \varphi_{H} \leq \varphi \leq \varphi_{G}, \\
x(\varphi, 0)=0,0 \leq y(\varphi, 0) \leq a, \varphi_{*} \leq \varphi \leq \varphi_{H}, \\
\left\{\begin{array}{l}
k_{21} \frac{\partial f_{i}}{\partial x} \frac{\partial x}{\partial \varphi}+k_{22} \frac{\partial f_{i}}{\partial y} \frac{\partial x}{\partial \varphi}-k_{11} \frac{\partial f_{i}}{\partial x} \frac{\partial y}{\partial \varphi}-k_{12} \frac{\partial f_{i}}{\partial y} \frac{\partial y}{\partial \varphi}=0, \\
f_{i}(x, y)=0, \quad i=1,3 \\
k_{11} \frac{\partial f_{i}}{\partial x} \frac{\partial y}{\partial \psi}-k_{12} \frac{\partial f_{i}}{\partial x} \frac{\partial x}{\partial \psi}+k_{21} \frac{\partial f_{i}}{\partial y} \frac{\partial y}{\partial \psi}-k_{22} \frac{\partial f_{i}}{\partial y} \frac{\partial x}{\partial \psi}=0, \\
f_{i}(x, y)=0, \quad i=2,4 \\
\frac{\partial}{\partial \varphi}\left(\frac{1}{\bar{k}} \frac{\partial x}{\partial \varphi}\right)+\underline{k} \frac{\partial}{\partial \psi}\left(\bar{k} \frac{\partial x}{\partial \psi}\right)+\left(k_{12}-k_{21}\right) \frac{\partial^{2} x}{\partial \varphi \partial \psi}=0, \\
\frac{\partial}{\partial \varphi}\left(\frac{1}{\bar{k}} \frac{\partial y}{\partial \varphi}\right)+\underline{k} \frac{\partial}{\partial \psi}\left(\bar{k} \frac{\partial y}{\partial \psi}\right)+\left(k_{12}-k_{21}\right) \frac{\partial^{2} y}{\partial \varphi \partial \psi}=0, \\
\bar{k} \frac{\partial f}{\partial J^{2}} \frac{\partial x}{\partial \varphi}\left(\frac{\partial y}{\partial \psi}\left(k_{21} \frac{\partial y}{\partial \psi}-k_{22} \frac{\partial x}{\partial \psi}\right)-\frac{\partial y}{\partial \psi}\left(k_{11} \frac{\partial y}{\partial \psi}-k_{12} \frac{\partial x}{\partial \psi}\right)\right), \\
s(x(\varphi, \psi), y(\varphi, \psi), 0)=\tilde{s}(x(\varphi, \psi), y(\varphi, \psi)), \tilde{s}\left(x\left(\varphi_{*}, \psi\right), y\left(\varphi_{*}, \psi\right)\right)=s_{0}, \\
0 \leq \psi \leq Q, \varphi_{*}<\varphi \leq \varphi^{*},
\end{array}\right.
\end{gathered}
$$

де $\underline{k}=k_{11} k_{22}-k_{21} k_{12}, \varphi_{C}, \varphi_{D}, \varphi_{G}, \varphi_{H}-$ значення квазіпотенціалу у відповідних точках. Тут умови (9) забезпечують ортогональність граничних та приграничних вузлів гідродинамічної сітки.

Різницевий аналог задачі будується аналогічно роботам [6-8], шляхом введення в області комплексного квазіпотенціалу рівномірної ортогональної сітки з вузлами в точках $\left(\varphi_{i}, \psi_{j}\right) \quad(i=\overline{1, n}, j=\overline{1, m})$, відносно якої здійснюється апроксимація рівнянь (8)-(11), наприклад, так:

$$
\begin{gathered}
x_{0, j}=r \cos \alpha, y_{0, j}=r \sin \alpha+b,-\frac{\pi}{2} \leq \alpha \leq \frac{\pi}{2}, j=\overline{0, m}, \\
x_{i, m}=0, b+r \leq y_{i, m} \leq 2 b, i=\overline{0, i_{C}}, \quad 0 \leq x_{i, m} \leq h, y_{i, m}=2 b, i=\overline{i_{C}, i_{D}}, \quad x_{i, m}=h, b+r \leq y_{i, m} \leq 2 b, i=\overline{i_{D}, n}, \\
x_{n, j}=r \cos \alpha+h, y_{n, j}=r \sin \alpha+b, \frac{\pi}{2} \leq \alpha \leq \frac{3 \pi}{2}, j=\overline{0, m}, \\
x_{i, 0}=h, 0 \leq y_{i, 0} \leq a, i=\overline{i_{G}, n}, 0 \leq x_{i, 0} \leq h, y_{i, 0}=0, i=\overline{i_{H}, i_{G}}, x_{i, 0}=0,0 \leq y_{i, 0} \leq a, i=\overline{0, i_{H}}, \\
\left\{\begin{array}{l}
k_{21} \frac{\partial f_{1}\left(x_{0, j}, y_{0, j}\right)}{\partial x}\left(x_{1, j}-x_{0, j}\right)+k_{22} \frac{\partial f_{1}\left(x_{0, j}, y_{0, j}\right)}{\partial y}\left(x_{1, j}-x_{0, j}\right)- \\
-k_{11} \frac{\partial f_{1}\left(x_{0, j}, y_{0, j}\right)}{\partial x}\left(y_{1, j}-y_{0, j}\right)-k_{12} \frac{\partial f_{1}\left(x_{0, j}, y_{0, j}\right)}{\partial y}\left(y_{1, j}-y_{0, j}\right)=0, \\
f_{1}\left(x_{0, j}, y_{0, j}\right)=0, \quad j=\overline{1, m-1},
\end{array}\right.
\end{gathered}
$$




$$
\begin{aligned}
& \left\{\begin{array}{l}
k_{21} \frac{\partial f_{3}\left(x_{n, j}, y_{n, j}\right)}{\partial x}\left(x_{n, j}-x_{n-1, j}\right)+k_{22} \frac{\partial f_{3}\left(x_{n, j}, y_{n, j}\right)}{\partial y}\left(x_{n, j}-x_{n-1, j}\right)- \\
-k_{11} \frac{\partial f_{3}\left(x_{n, j}, y_{n, j}\right)}{\partial x}\left(y_{n, j}-y_{n-1, j}\right)-k_{12} \frac{\partial f_{3}\left(x_{n, j}, y_{n, j}\right)}{\partial y}\left(y_{n, j}-y_{n-1, j}\right)=0 \\
f_{3}\left(x_{n, j}, y_{n, j}\right)=0, \quad j=\overline{1, m-1},
\end{array}\right. \\
& \left\{\begin{array}{l}
k_{11} \frac{\partial f_{2}\left(x_{i, m}, y_{i, m}\right)}{\partial x}\left(y_{i, m}-y_{i, m-1}\right)-k_{12} \frac{\partial f_{2}\left(x_{i, m}, y_{i, m}\right)}{\partial x}\left(x_{i, m}-x_{i, m-1}\right)+ \\
+k_{21} \frac{\partial f_{2}\left(x_{i, m}, y_{i, m}\right)_{i}}{\partial y}\left(y_{i, m}-y_{i, m-1}\right)-k_{22} \frac{\partial f_{2}\left(x_{i, m}, y_{i, m}\right)}{\partial y}\left(x_{i, m}-x_{i, m-1}\right)=0 \\
f_{2}\left(x_{i, m}, y_{i, m}\right)=0, \quad i=\overline{1, n-1}
\end{array}\right. \\
& \left\{\begin{array}{l}
k_{11} \frac{\partial f_{4}\left(x_{i, 0}, y_{i, 0}\right)}{\partial x}\left(y_{i, 1}-y_{i, 0}\right)-k_{12} \frac{\partial f_{4}\left(x_{i, 0}, y_{i, 0}\right)}{\partial x}\left(x_{i, 1}-x_{i, 0}\right)+ \\
+k_{21} \frac{\partial f_{4}\left(x_{i, 0}, y_{i, 0}\right)_{i}}{\partial y}\left(y_{i, 1}-y_{i, 0}\right)-k_{22} \frac{\partial f_{4}\left(x_{i, 0}, y_{i, 0}\right)}{\partial y}\left(x_{i, 1}-x_{i, 0}\right)=0 \\
f_{4}\left(x_{i, 0}, y_{i, 0}\right)=0, \quad i=\overline{1, n-1},
\end{array}\right. \\
& \gamma^{2} \underline{k}\left(\bar{k}_{i, j+1 / 2}\left(x_{i, j+1}-x_{i, j}\right)-\bar{k}_{i, j-1 / 2}\left(x_{i, j}-x_{i, j-1}\right)\right)+\gamma\left(k_{12}-k_{21}\right)\left(x_{i+1, j+1}-x_{i+1, j}-x_{i, j+1}+x_{i, j}\right)+ \\
& +\frac{x_{i+1, j}-x_{i, j}}{\bar{k}_{i+1 / 2, j}}-\frac{x_{i, j}-x_{i, j-1}}{\bar{k}_{i-1 / 2, j}}=0, \\
& \gamma^{2} \underline{k}\left(\bar{k}_{i, j+1 / 2}\left(y_{i, j+1}-y_{i, j}\right)-\bar{k}_{i, j-1 / 2}\left(y_{i, j}-y_{i, j-1}\right)\right)+\gamma\left(k_{12}-k_{21}\right)\left(y_{i+1, j+1}-y_{i+1, j}-y_{i, j+1}+y_{i, j}\right)+ \\
& +\frac{y_{i+1, j}-y_{i, j}}{\bar{k}_{i+1 / 2, j}}-\frac{y_{i, j}-y_{i, j-1}}{\bar{k}_{i-1 / 2, j}}=0, \\
& \bar{s}_{i, j}=s_{i, j}-\frac{\bar{\tau}_{i, j}\left(f\left(s_{i, j}\right)-f\left(s_{i-1, j}\right)\right)}{\sigma \Delta \varphi \Delta \psi^{2} J_{i, j}^{2}} \\
& \times\left(k_{11}\left(y_{i, j}-y_{i, j-1}\right)^{2}+k_{22}\left(x_{i, j}-x_{i, j-1}\right)^{2}-\left(k_{12}+k_{21}\right)\left(y_{i, j}-y_{i, j-1}\right)\left(x_{i, j}-x_{i, j-1}\right)\right), \\
& s\left(x_{i, j}, y_{i, j}, 0\right)=\tilde{s}\left(x_{i, j}, y_{i, j}\right), \tilde{s}\left(x_{0, j}, y_{0, j}\right)=s_{0}, i=\overline{1, n-1}, j=\overline{1, m-1}, \\
& \text { де } \quad \bar{k}_{i, j \pm 1 / 2}=\frac{\bar{k}_{i, j \pm 1}+\bar{k}_{i, j}}{2}, \quad \bar{k}_{i \pm 1 / 2, j}=\frac{\bar{k}_{i \pm 1, j}+\bar{k}_{i, j}}{2}, \quad \gamma=\frac{\Delta \varphi}{\Delta \psi}, \quad x_{i, j}=x\left(\varphi_{i}, \psi_{j}\right), \quad y_{i, j}=y\left(\varphi_{i}, \psi_{j}\right) \text {, }
\end{aligned}
$$$$
\bar{k}_{i, j}=\bar{k}\left(x_{i, j}, y_{i, j}, s_{i, j}\right), \tau \text { - крок по часу, } J_{i, j}=\frac{\left(x_{i, j}-x_{i-1, j}\right)\left(y_{i, j}-y_{i, j-1}\right)-\left(y_{i, j}-y_{i-1, j}\right)\left(x_{i, j}-x_{i, j-1}\right)}{\Delta \varphi \Delta \psi},
$$

$s_{i, j}, \bar{s}_{i, j}$ - насиченості у відповідних вузлах $(i, j)$ на попередньому та наступному кроці ітерації по часу. Кроки алгоритму розв'язання різницевої задачі співпадають з кроками алгоритму, що описаний в роботі [6], для випадку ізотропного пласта, за умови підстановки в них рівнянь (12)-(15).

Комп'ютерний експеримент. На рис. 2-5 зображено гідродинамічні сітки та розподіл насиченості в пласті при $\varphi_{*}=0, \varphi^{*}=1, n=30, m=20, \varepsilon=0.0001, \sigma=0.5, \tilde{k}_{o}(s)=(1-s)^{2}$, 
$\tilde{k}_{w}(s)=s^{2}, \mu_{o}=2, \mu_{w}=1, \tau=0.01$ та різних значеннях тензора абсолютної проникності в момент часу $t=21$.

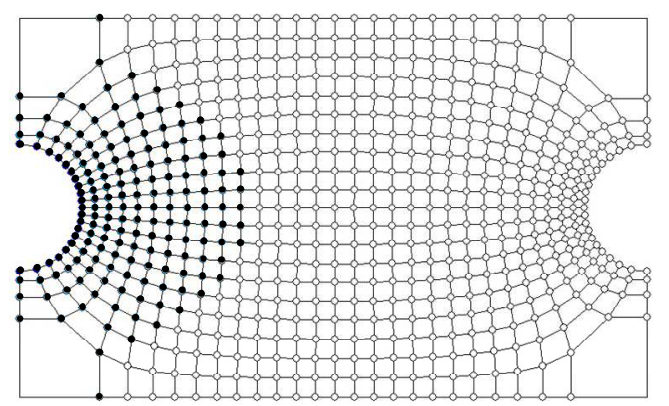

Puc. 2. Гідродинамічна сітка та розподіл насиченості в пласті при $k=\left(\begin{array}{ll}1 & 0 \\ 0 & 1\end{array}\right)$.

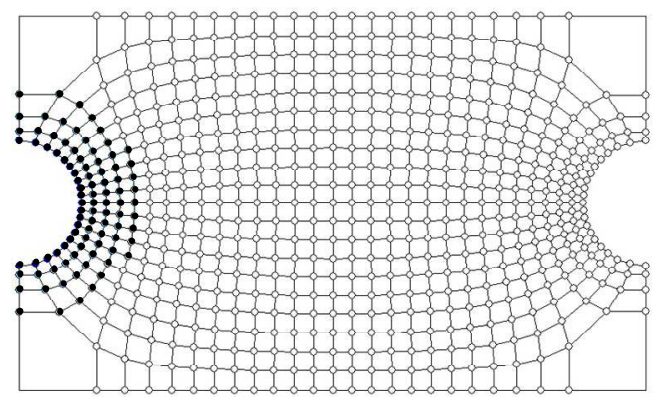

Рuc. 3. Гідродинамічна сітка та розподіл насиченості в пласті при $k=\left(\begin{array}{cc}0.3 & 0 \\ 0 & 0.3\end{array}\right)$.

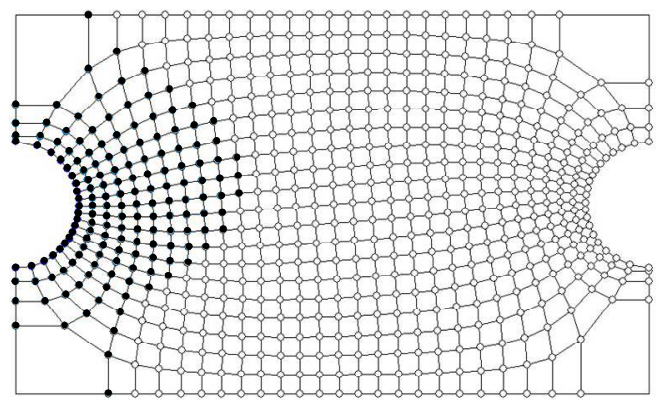

Рuc. 4. Гідродинамічна сітка та розподіл насиченості в пласті при $k=\left(\begin{array}{cc}1 & 0 \\ 0.5 & 1\end{array}\right)$.

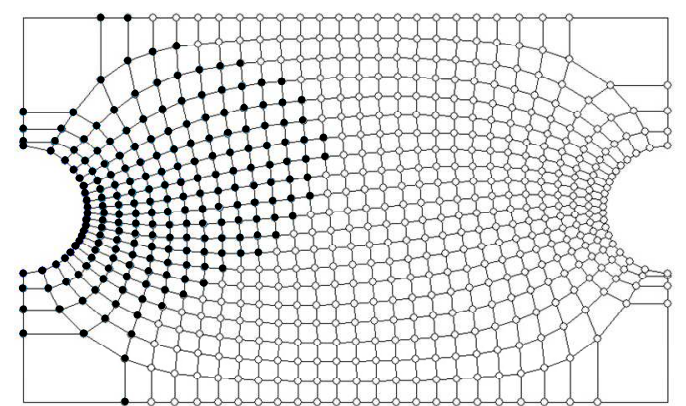

Рuc. 5. Гідродинамічна сітка та розподіл насиченості в пласті при $k=\left(\begin{array}{ll}1.4 & 0.5 \\ 1.3 & 1.6\end{array}\right)$. 


\section{Висновки}

В роботі розвинуто числові методи комплексного аналізу на випадок дослідження двофазної фільтрації в анізотропних грунтах. 3 використанням системи типу Коші-Рімана (у випадку врахування анізотропії), побудовано нові умови для визначення положення граничних вузлів гідродинамічної сітки відносно приграничних. 3 урахуванням цього було модифіковано відповідний алгоритм числового квазіконформного відображення та здійснено його програмну реалізацію. Сформовано відповідні крайові задачі та здійснено їх розв'язання. Проведено ряд числових експериментів, впливу зміни компонент тензора проникності на напрямок течії в елементі симетрій рядної системи площового заводнення пласта. Та помічено, що у випадках не симетрії тензора формуються застійні зони, для уникнення яких, на нашу думку, потрібно провести процедуру гідравлічного розриву пласта.

\section{Список використаної літератури}

1. Басниев К. С., Кочина И. Н., Максимов В. М. Подземная гидромеханика. М. : Наука, 1993. $125 \mathrm{c}$.

2. Баренблатт Г.И., Ентов В. М., Рыжик В. М. Теория нестационарной фильтрации жидкости и газа. М. : Недра, 1972. 288 с.

3. Пергамент А. Х., Томин П. Ю. Об исследовании функций относительных фазовых проницаемостей для анизотропных сред. Матем. моделирование, 2011. Т. 23, № 5. С. 3-15.

4. Толпаев В.А., Мартынов В.В., Евенко И.А. Задачи фильтрации жидкости к вертикальной скважине в пласте с прямолинейной анизотропией. Автоматизация, телемеханизация и связь в нефтяной промышленности, 2008. № 5. С. 47-54.

5. Фазлыев Р. Т. Площадное заводнение нефтяных месторождений. М. : Ижевск, ИКИ, НИЦ РХД, 2008. $256 \mathrm{c}$.

6. Бомба А. Я., Каштан С. С., Пригорницький Д. О., Ярощак С. В. Методи комплексного аналізу. Рівне : НУВГП, 2013. 415 с.

7. Бомба А. Я., Сінчук А. М. Комплексний аналіз поведінки системи «свердловини-тріщинипласт» в елементах площадного заводнення. Вісник НТУ «ХПІ». Серія «Математичне моделювання в техніці та технологіях». Харків: НТУ «ХПІ», 2013. № 54(1027). С.4-15.

8. Бомба А. Я., Синчук А. М., Ярощак С. В. Метод комплексного анализа исследования двухфазной фильтрации в горизонтальных пластах с учетом гидроразрыва. Электронное моделирование. 2013. Т. 35, № 2. С. 25-33.

\section{MATHEMATICAL MODELING OF TWO-PHASE FILTRATION IN ANISOTROPIC OIL LAYERS BY QUASIC-FORMAL MAPPING Sinchuk A.}

\section{Abstract}

Oil production has been deteriorating over the last decades. Whereas the process of filtration of different types of liquids (water, oil etc.) occurs in the porous medium, which, due to their physical and mechanical properties, belong to the group of isotropic or anisotropic soils. Heterogeneous anisotropic media are more complicated by their structure. Their high heterogeneity, in particular, leads to an uneven oil extraction, the reduction of oil recovery coefficient, etc. Therefore, there is a problem of nonlinear filtration modeling in anisotropic media.

Most existing models describe the filtration processes under the conditions of the layer isotropy, when the permeability coefficient is a scalar function [1,2]. However, in practice, there are layers whose permeability coefficient depends on the filtration direction. That is, the pressure gradient is not collinear to the velocity vector. In such cases, there is the phenomenon of layer anisotropy [3-5], and the Darcy law, with the compatible motion of several liquids, acquires different look. 
According to this, there is a need to summarize the numerical methods of complex analysis [6-8], that have been developed, in case of anisotropy layer formation.

Using the Cauchy-Riemann-type system (in the case of anisotropy), new conditions have been constructed to determine the position of the boundary nodes of the hydrodynamic grid relatively to cross-border ones. In view of this, the corresponding algorithm of the numerical quasi-conformal mapping was modified and its software implementation was realized. The corresponding boundaryvalue problems were formed and their solutions were solved. A series of computer experiments on the effect of changing the permeability tensor components on the direction flows in the symmetry element of the in-line system of the layer flooding was carried out. Consequently, it is noted that, in cases, where the tensor is not symmetry, the stagnant zones are formed. To avoid this, in our opinion, they should be performed by the procedure of hydraulic fracturing of the layer.

In the future it is planned to use the numerical algorithm of integrated analysis methods, presented in the paper, to account the impact of fractures on the wells in oil production.

\section{References}

1. Basniev, K.S., Cochina, I.N., Maksimov, V.M. (1993) Podzemnaya gidromekhanika. M.: Nedra, 125.

2. Barenblatt, G.I., Entov, V.M., Ryzhik, V.M. (1972) Theory of unsteady liquid and gas filtration. M.: Nedra, 288.

3. Parchment, A. Kh., Tomin, P. Yu. (2011) Yu. On the study of relative phase permeability functions for anisotropic media. Mathematical Modeling, T. 23, № 5, 3-15.

4. Tolpaev, V.A., Martynov, V.V., Evenko, I.A.(2008) The tasks of filtering fluid to a vertical well in a reservoir with rectilinear anisotropy. Automation, telemechanization and communications in the oil industry, № 5, 47-54.

5. Fazlyev, R. T. (2008) Area flooding of oil fields. M.: Izhevsk, IKI, SIC RHD, 256.

6. Bomba, A.Ya., Kashtan, S.S., Prigornitsky, D.O., Yaroshchak, S.V. (2013) Methods of complex analysis. Rivne: NUVGP, 415.

7. Bomba, A.Ya., Sinchuk, A. M. (2013) A comprehensive analysis of the behavior of the "drill-pipepipe-system" system in the elements of areal waterflooding. Newsletter NTU "KhPI". Seriya "Mathematical modeling in technology and technology." Kharkiv: NTU "KhPI", № 54(1027), 415.

8. Bomba, A.Ya., Sinchuk, A. M., Yaroshchak, S.V. (2013) The method of complex analysis of the study of two-phase filtration in horizontal layers taking into account hydraulic fracturing. Electronic modeling, T. 35, № 2, 25-33. 